\title{
Production of yellow fever 17DD vaccine virus in primary culture of chicken embryo fibroblasts: yields, thermo and genetic stability, attenuation and immunogenicity
}

\author{
Marcos S. Freire $^{\text {a }}$, George F. Mann ${ }^{\mathrm{a}, *, 1}$, Renato S. Marchevsky ${ }^{\mathrm{a}}$, Anna M.Y. Yamamura ${ }^{\mathrm{a}}$, \\ Luiz F.C. Almeida ${ }^{\mathrm{a}}$, Alfredo V. Jabor ${ }^{\mathrm{c}}$, José M.N. Malachias ${ }^{\mathrm{a}}$, \\ Evandro S.F. Coutinho ${ }^{\mathrm{b}}$, Ricardo Galler ${ }^{\mathrm{c}}$ \\ a Fundacao Oswaldo Cruz, Instituto de Tecnologia em Imunobiológicos, Avenida Brasil 4365, \\ Manguinhos, Rio de Janeiro 21045-900, Brazil \\ b Escola Nacional de Saude Pública, Rio de Janeiro, RJ, Brazil \\ c Instituto Oswaldo Cruz, Rio de Janeiro, RJ, Brazil
}

Received 26 April 2004; received in revised form 4 October 2004; accepted 8 October 2004

Available online 28 November 2004

\begin{abstract}
While a good vaccine against yellow fever (YF) virus has been available for decades, the basic technology for the production of YF vaccine in chicken embryos has remained substantially unchanged since the 1940s. Here we describe the highly efficient and economic production of the 17DD strain of YF virus in chicken embryo fibroblast (CEF) cell cultures with viral titers ranging from 6.3 to $6.7 \log _{10} \mathrm{PFU} / \mathrm{mL}$. Thermostability of two different formulations (5 and 50-dose vials) of the CEF vaccine virus was found to be as high as the current vaccines retaining the minimal titer required for YF 17D vaccines. The production passage in CEF did not lead to the selection of genetic variants as shown by nucleotide sequence analyses of the CEF-derived vaccine lots or the sequence of viruses recovered from monkeys experimentally inoculated with the CEF virus. YF 17DD virus produced in CEF was also indistinguishable from its seed lot virus parent in terms of plaque size and immunogenicity in mice and monkeys. Comparison of the CEF virus and the seed lot virus made in chicken embryo in the internationally accepted monkey neurovirulence test (MNVT) revealed a higher clinical score for the former. The differences in central nervous system (CNS) histological scores for monkeys inoculated with the chicken embryo and experimental CEF vaccines were at the borderline level of statistical significance. These data warrant further studies on the monkey attenuation of other batches of CEF-derived vaccines.
\end{abstract}

(C) 2004 Elsevier Ltd. All rights reserved.

Keywords: Yellow fever; 17D virus; Primary culture; Vaccine; Attenuation

\section{Introduction}

While a good vaccine against YF has been available since the late 1930s the basic technology for its production in chicken embryos has remained substantially unchanged. Some improvements, such as elimination of the avian leucosis virus complex and increased thermostability have been

\footnotetext{
* Corresponding author. Tel.: +55 213882 9317; fax: +55 2122604727.

E-mail address: freire@bio.fiocruz.br (G.F. Mann).

${ }^{1}$ In memoriam.
}

made in recent years [1]. This vaccine nevertheless, still contains more foreign protein than any other live virus vaccine (up to $250 \mu \mathrm{g}$ of chicken embryo protein per human dose) and the need for large numbers of whole eggs, and large amounts of seed virus to inoculate these eggs introduce limitations into expanding production of this important vaccine. Therefore, new production technology would be important to provide an improved product on a sufficient scale to meet the demands of routine vaccination in endemic areas and the control of potential epidemic situations. 
The 17DD yellow fever vaccine has been produced at the Oswaldo Cruz Foundation since 1936. The current whole chicken embryo product meets all World Health Organization (WHO) requirements [2] and is free of the avian leucosis virus complex [3]. A meeting was held in the Pan American Health Organization (PAHO) to develop guidelines for the production of yellow fever vaccine in cell cultures [4]. Following this meeting, work on the development of a cell culture vaccine was initiated in Bio-Manguinhos. By 1988 a cell culture vaccine had been produced and tested with satisfactory results in all assays including monkey neurovirulence test (MNVT) $[5,6]$. Subsequently, this process was found to be not feasible since large amounts of costly seed virus were required to obtain acceptable yields. Developmental studies were restarted in 1996 and here we describe the economically and efficient production of YF 17DD virus in primary cultures of chicken embryo fibroblasts (CEF) and describe the phenotypic and molecular characteristics of the resulting virus.

\section{Materials and methods}

\subsection{Cells}

Vero cells (ATCC, CCL 81) were maintained in Medium 199 with Earle's salts (E199), buffered with sodium bicarbonate and supplemented with 5\% fetal bovine serum (FBS) and antibiotics.

\subsection{Preparation of chicken embryo fibroblast cultures and production of experimental vaccine lots of YF $17 D D$ virus}

Eleven-day-old chicken embryos are removed from specific pathogen free (SPF) eggs and the head and viscera dissected out. The tissue is then washed with PBS, extruded and treated with trypsin to give a monodisperse cell suspension [7]. Cells are then seeded into roller bottles in 199 medium with FBS. This serum was obtained from Cultilab (Brazil), it was not irradiated but heat-inactivated prior to use. Its quality control was performed by our QC Department and included several adventious agents. Bottles were incubated at $37^{\circ} \mathrm{C}$ with rotation at $15 \mathrm{rph}$. After a $24 \mathrm{~h}$ incubation, medium is removed and the cells infected with the 102/84 seed virus used for production of 17DD vaccine in eggs at constant multiplicity of infection (moi) of 0.002 by adsorption at $37^{\circ} \mathrm{C}$ for $1 \mathrm{~h}$. Following the adsorption, bovine serum concentration was reduced to $<1$ part per million by washing the CEF monolayers twice with Earle's BSS prior to addition of 199 medium without FBS. Virus is harvested at $48 \mathrm{~h}$ post infection, clarified and frozen to $-70^{\circ} \mathrm{C}$. Viruses were recovered by pooling the medium present in every three roller into a centrifuge tube and spinning at low speed to remove cellular debris. The supernatant was aspirated into flasks containing a stabilizer formulation at a 1:1 ratio and frozen by rotating on an ethanol dry ice bath after removal of all quality control aliquots. The same stabilizer used to formulate the five-dose commercial egg-produced YF 17DD vaccine was also added to the cell culture-produced virus. It consists of hydrolysed gelatin, sugar and amino acids. All viruses are stored as indicated. Quality control tests for this product have been conducted according to WHO minimal requirements for mumps [8] and other vaccines produced in chicken embryo cells in addition to those tests specific to yellow fever [2].

\subsection{Viruses}

The passage history of YF 17DD strain and the derivation of the 102/84 seed lot has been previously described [9]. The YF 17DD vaccine lots used in this study are licensed for human immunization and the experimental vaccine lots produced in CEF (YFCEF-01-07) cultures are at passage level 286. The 102/84 seed virus present in the original vials and at passage level 285 was reconstituted in $0.5 \mathrm{~mL}$ of sterile water as specified by the manufacturer (FIOCRUZ/BioManguinhos, Rio de Janeiro, Brazil) and used to inoculate primary CEF cultures as described above (moi of 0.002).

Viruses recovered from monkey sera (R5 and Q15) were passaged once in Vero cells at a multiplicity of infection (moi) of 0.1-1.0 PFU/cell and cell culture supernatant harvested at 3-4 days post infection (p.i.). There was extensive cytopathic effect (CPE) on the cell monolayers and the supernatants were the source of virus for RNA extraction. Virus titration and plaque size determination were carried out on Vero cells as described elsewhere [10].

\subsection{Thermostability assay}

Three freeze-dried vials of each experimental batch and the control vaccine were incubated at $37^{\circ} \mathrm{C}$ for 2 weeks. The virus concentrations were determined using virus plaquing on Vero cells having as control the unheated vaccine. The average titers of the three vials exposed or not to the test temperature were used to calculate thermodegradation.

\subsection{Neutralization assay}

Antibody titers were determined by $50 \%$ plaque reduction neutralization test on Vero cells. For monkey sera the plaque reduction neutralization test (PRNT) was conducted in serial four-fold dilutions starting at 1:16 in six-well tissue culture plates [11]. For mice, PRNT assays were carried out on Vero cells in 96-well plates, as described elsewhere [12]. Results were also expressed in milli international units per milliliter $(\mathrm{mIU} / \mathrm{ml})$ using a reference preparation of monkey yellow fever serum containing 14,300 mIU/ml (Copenhagen Serum Institute). Although this unit system is not frequently used in the literature to express yellow fever neutralizing antibody titers, we provide the data as such to enable our results to be compared with those from other laboratories using a standardized unit system. 


\subsection{Complementary DNA synthesis, amplification and sequencing}

Virus samples from test monkeys were obtained during viremia periods and passaged once in Vero cell cultures for amplification of virus titers (see above). Total RNA was extracted from the media of infected cell cultures with TRIzol ${ }^{\circledR}$ LS reagent (Invitrogen) using $20 \mu \mathrm{g}$ of glycogen as carrier. RNA of the inoculum virus was obtained directly from the original test inoculum, which was diluted from CEF cultures to a titer of $100,000 \mathrm{PFU} / \mathrm{mL}$.

Complementary DNA synthesis (RT) and amplification (PCR) were performed using a GeneAmp RNA PCR kit and a GeneAmp 9600 instrument (Applied Biosystems). RT-PCR amplification of viral genomic regions was performed essentially as described previously [13]. A total of 11 RT-PCR fragments were designed comprising nucleotides 1-1066, 940-1799, 1641-2639, 2361-3387, 3004-4286, 4181-5071, 4980-6325, 6102-7272, 7162-8420, 8302-9586 and 9425-10862, within which numerous internal primers were positioned on both cDNA strands for the overlapping sequencing reactions. Nucleotides were numbered and primers were designed after the yellow fever 17D vaccine strain sequence ([14]; GenBank accession no. X03700, formerly no. K02749). Amplification products were further purified from excess primers with silica-based purification kits (QIAGEN). The purified products were sequenced directly without molecular cloning. Nucleotide sequencing reactions were performed with the BigDye terminator mix version 2.0 (Applied Biosystems) according to the manufacturer's recommendations. Electrophoresis of fluorescent products was performed in an ABI PRISM 3100 instrument (Applied Biosystems). Nucleotide sequences were analyzed using Chromas software version 1.45 (Technelysium) and a consensus sequence for each virus genome was derived from contiguous sequences using SeqMan II software from Lasergene package version 4.05 (DNAStar).

\subsection{Animal studies}

Studies were conducted using a protocol approved by the Institutional Committee of Animal Care and Experimentation (CEUA-FIOCRUZ: P0112/02).

\subsubsection{Mice}

Groups of 3-week-old Swiss Webster mice were inoculated by the intracerebral (i.c.) route with serial 10-fold dilutions (in $30 \mu \mathrm{L}$ ) of YF 17DD vaccine (lot number 035VFA035P), YFCEF-03, R5 and Q15 viruses. Viruses were diluted in complete 199 culture medium and the inoculum was back-titered immediately after the challenge procedure. Animals were monitored for 21 days and deaths recorded. Moribund animals were euthanized by exposure to carbon dioxide. The $\mathrm{MLD}_{50}$ [15] was calculated taking into account the cumulative death rates for each dilution that together with the titers in PFU originated from back titration to establish the amount of virus (in PFU) required to kill $50 \%$ of the mice. Linear regression analysis was used to infer the $50 \%$ end-point.

Kaplan-Meier curves were drawn and log rank test was used to calculate the statistical significance of the difference between the groups. The Kruskal-Wallis non-parametric test was used to analyze the significance of difference in average survival times. All tests above were done using Stata 7.0 software (Stata Corp., College Station, Texas, 2002).

For immunogenicity studies, 3-week-old Swiss Webster mice (Mus musculus) were immunized by the subcutaneous (s.c.) route with one dose of $0.1 \mathrm{~mL}$ of complete 199 culture medium containing $10^{5} \mathrm{PFU}$ of YF 17DD virus. Mice were bled from the retro-orbital plexus before immunization and twice after immunization with 2 -week intervals. Neutralizing antibody titers were determined on individual sera. Challenge was carried out 45 days after immunization by i.c. inoculation of YF17DD vaccine virus (lot number 035VFA035P). Mortality and average survival time were scored for 21 days.

\subsubsection{Monkeys}

Two groups of 10 captive-bred healthy rhesus monkeys (Macaca mulatta), 12 male and eight female, weighing 2430-3600 g were obtained from the Primatology Department of the Breeding Center for Laboratory Animals of the Oswaldo Cruz Foundation (FIOCRUZ), Rio de Janeiro, RJ, Brazil. Each animal was kept in a separate cage under controlled environmental conditions (temperature $20-22^{\circ} \mathrm{C}$, relative humidity $\sim 60 \%$ and $12 \mathrm{~h}$ of artificial light and $12 \mathrm{~h}$ of darkness). Animals were fed twice daily with monkey chow supplemented with fresh fruits and allowed water ad libitum. All monkeys were shown to be free of YF neutralizing antibodies by PRNT assay. Virus inoculation, viremia, seroconversion, clinical observation, necropsy and histological examination have been previously described $[11,13]$.

\subsubsection{Virus inoculation}

All monkeys were anaesthetized by intramuscular injection of ketamine hydrochloride $(20 \mathrm{mg} / \mathrm{kg}$ body weight) and inoculated with $0.25 \mathrm{~mL}$ of viral suspension by the intracerebral (i.c.) route into the right frontal cortex by making an incision through the skin about $2.5 \mathrm{~cm}$ above the middle of the right superior orbital ridge and a hole was drilled through the skull. Virus solution was inoculated into the frontal lobe using a $1 \mathrm{~mL}$ syringe fitted with a 25 -gauge $\times 5 / 8$ needle.

The viral inocula were back titrated by plaque assay on Vero cells. Yellow Fever secondary seed lot (YFV 17DD 102/84) dose was 24,807 mouse $\mathrm{LD}_{50} / 0.25 \mathrm{~mL}$. YF 17DD CEF03 virus dose was 16,080 mouse $\mathrm{LD}_{50} / 0.25 \mathrm{~mL}$.

\subsubsection{Viremia}

Blood samples for the determination of viremia were collected on days 2, 4 and 6 after inoculation. Serial dilutions $(1: 3,1: 30,1: 300)$ of each monkey serum were titered by plaque assay on Vero cell monolayers $\left(10^{5}\right.$ cells $\left./ \mathrm{cm}^{2}\right)$ with $3.5 \%$ carboxymethylcellulose as overlay in six-well plates. 
One hundred microliters of virus suspension was inoculated per well with four-well per dilution.

\subsubsection{Seroconversion}

Antibody titers were determined by a 50\% plaque reduction test on Vero cells (Stefano et al. [12]).

\subsubsection{Clinical observation}

Monkeys were observed for 30 days and rectal temperature recorded daily. Temperature equal or greater than $40.0^{\circ} \mathrm{C}$ was considered elevated. Records of clinical observation were obtained using the following signs: grade $1=$ rough coat, not eating; grade 2 = high-pitched voice, inactive, slow moving; grade $3=$ shaky movements, tremors, uncoordinated movement, limb weakness; grade 4 = inability to stand, limb paralysis or death. A monkey that dies receives the score " 4 " from the day of death until day 30 .

\subsubsection{Necropsy and histological examination}

All 20 animals were submitted to full necropsy at the end of the observation period. They were sacrificed by exsanguination under deep anaesthesia. Serum samples were collected to determine anti-YFV antibodies by plaque-reduction neutralization-assay.

Five levels of the brain and six levels of each of the lumbar and cervical enlargements were examined. Brain levels included: block I, the corpus striatum at the level of the optic chiasma; block II, the thalamus at the level of the mamillary bodies; block III, the mesencephalon at the level of the superior colliculi; block IV, the pons and cerebellum at the level of the superior olives; block V, the medulla oblongata at the mid-level of the inferior olives.

Numerical scores were given to each hemisection of the cord and to structures in each hemisection of the brain. Lesions were scored according to the following grading system: (1) (minimal), 1-3 small, focal inflammatory infiltrates, a few neurons may be changed or lost; (2) (moderate), more extensive focal inflammatory infiltrates, neuronal changes or loss affects no more than one-third of neurons; (3) (severe), neuronal changes or loss of 33-90\% of neurons, with moderate focal or diffuse inflammatory infiltration; (4) (overwhelming), more than $90 \%$ of neurons are changed or lost, with variable, but frequently severe, inflammatory infiltration.

Three separate scores were calculated for each monkey: discriminator areas only, target areas only, and discriminator plus target areas Levenbook et al. [27]. The target area is the substantia nigra whereas the discriminator areas include the caudate nucleus, globus pallidus, putamen, anterior and medial thalamic nucleus, lateral thalamic nucleus, cervical and lumbar enlargements. A final neurovirulence score is given by the combination of the scores of both areas (combined score).

Overall mean scores were calculated for each group of monkeys as the arithmetic mean of individual monkey scores for discriminator areas only and for discriminator plus target areas. For the histological criterion of the neurotropism test to be satisfied both overall mean scores for the test monkeys shall not be significantly greater (at the 5\% significance level) than the overall mean scores for the monkeys injected with reference virus.

\section{Results}

\subsection{Production of $Y F$ 17DD virus in primary $C E F$ cultures}

Despite the advantages of using certified SPF-CEF cells to produce vaccine viruses, such as YF, measles and mumps, it is frequently found that the process is relatively uneconomical due to low yields [1]. This is particularly true for the YF vaccine virus produced in CEF at the cell densities normally used $\left(1 \times 10^{6}\right.$ cells $\left./ \mathrm{cm}^{2}\right)$ where yield is directly related to seed virus input. Thus, the amount and cost of the seed virus necessary under these conditions is too high especially in light of the required monkey neurovirulence test needed for each seed lot.

To overcome the limited yields in the CEF cultures we investigated the consequences of cellular density at the time of viral infection on viral yields. The results of a typical experiment to test the effect of cell seeding density on the yield of YF 17DD virus from CEF using a constant moi of $0.00224 \mathrm{~h}$ after cell seeding, are presented in Table 1.

These results unequivocally demonstrate that the $\log _{10}$ virus yield is inversely and linearly related to the $\log _{10}$ concentration $(r=0.994, P<0.001)$ of cells seeded in the range of $3 \times 10^{4}$ to $2 \times 10^{5}$ cells $/ \mathrm{cm}^{2}$. In this range, a $2.2 \log _{10}$ increase in virus yield/mL is obtained for each $\log _{10}$ decrease in the cell concentration. A concentration of $3 \times 10^{4}$ cells $/ \mathrm{cm}^{2}$ in which the maximal yield per milliliter was obtained, the yield per cell was $250 \mathrm{PFU} /$ cell or approximately 400-fold more than that obtained at $2 \times 10^{5}$ cells $/ \mathrm{cm}^{2}$. These results definitively demonstrate the infectious virus yield/mL and yield/cell are inversely related to the cell seeding density. Thus, efficient production can be established. We have tested two other YF 17D substrains, 17D-213 [16] and a complementary DNA-derived virus YFiv5.2/VL [17] and they behaved in the same manner (data not shown).

Table 1

Influence of cell seeding density on the yield of YF 17D virus from CEF

\begin{tabular}{llc}
\hline Cells/cm & \multicolumn{2}{l}{ Yields } \\
\cline { 2 - 3 } & $\log _{10}$ PFU/mL & PFU/cell \\
\hline 10000 & 7.11 & 513 \\
30000 & 7.28 & 251 \\
50000 & 6.78 & 47.9 \\
70000 & 6.30 & 11.5 \\
90000 & 6.15 & 6.3 \\
200000 & 5.48 & 0.6
\end{tabular}

${ }^{a}$ Density at time of infection. 
Table 2

Yields of yellow fever 17DD vaccine produced in primary CEF cultures under Good Manufacturing Practices

\begin{tabular}{ll}
\hline Batch $^{\mathrm{a}}$ & Titer $\left(\log _{10} \mathrm{PFU} / \mathrm{mL}\right)$ \\
\hline YFCEF-01 & 6.79 \\
YFCEF-02 & 6.68 \\
YFCEF-03 & 6.66 \\
YFCEF-04 & 6.75 \\
YFCEF-05 & 6.32 \\
YFCEF-06 & 6.69 \\
YFCEF-07 & 6.32 \\
\hline
\end{tabular}

${ }^{a}$ Each batch was tested for sterility, potency and adventitious agents with satisfactory results.

In order to show reproducibility of YF 17DD virus propagation and yields in CEF cultures a total of 21 roller bottles with a surface area of $680 \mathrm{~cm}^{2}$ each was used for each of the seven experimental batches. The batch size was calculated to provide enough volume to perform all laboratory and pre-clinical tests and eventually a phase I clinical trial. The yields are presented in Table 2. Titers varied from 6.32 to $6.79 \log _{10} \mathrm{PFU} / \mathrm{mL}$ after the addition of stabilizer. Assuming a dose of $4.3 \log _{10}$ PFU the average batch size is approximately 400,000 doses of YF 17DD vaccine.

\subsection{Thermostability analysis of $Y F 17 D D$ virus}

We have shown above that YF 17D vaccine virus production in primary CEF cultures is feasible from the production standpoint. We have next asked whether virus produced accordingly would also retain the thermostability properties characteristic and required for YF 17D vaccines produced in chicken embryos according to current manufacturing process. Table 3 shows the results of three different rounds of filling and freeze-drying using three batches of virus concentrate (5-7; Table 2) and two different formulations (5 and 50-dose vials).

According to the Requirements for Yellow Fever Vaccine [2] the freeze-dried vaccine shall not loose more than $1 \log _{10}$ PFU per dose and keep the titer higher than 1000 mouse LD $_{50}$ or the equivalent number of PFU/dose after exposure to $37^{\circ} \mathrm{C}$. To facilitate evaluation of thermal stability, we established an in-house plaque assay, and demonstrated a ratio of PFU/LD ${ }_{50}$ of $0.7 \log _{10}$. In the three experiments the commercial vaccine showed losses of 0.6, 0.47 and $0.52 \log _{10}$ PFU/dose. The experimental vaccines with five-dose formulation lost between 0.18 (experiment 02 ) and $0.39 \log _{10}$ PFU/dose (experiment 03 ), and the 50-dose presentation showed losses between 0.45 (experiment 02) and $0.57 \log _{10} \mathrm{PFU} / \mathrm{dose}$ (experiment 01). Thus, all of the lots of experimental CEF-propagated vaccine that we tested by these methods achieved a PFU/dose titer of at least $3.78 \log _{10}$ PFU/dose, corresponding to $3.08 \log _{10} \mathrm{MLD}_{50} /$ dose, a level that exceeds the internationally established minimal dose requirement.

\subsection{Genetic stability}

During the production process there is a tremendous amplification of viral RNA to form new viral particles allowing for selection of viruses with altered genomes [18]. Although Pugachev et al. [19] has recently suggested a high degree of genetic stability of YF virus under these conditions we have examined whether the propagation of the 17DD 102/84 seed lot virus in CEF led to the selection of any genetic variants. For this purpose we sequenced the genome of the YFCEF-03 (Table 2) virus and of two viruses recovered from monkeys R5 and Q15 (see Table 6) which received YFCEF-03 virus in the MNVT. No nucleotide sequence differences were observed between the original 102/84 seed virus and the YFCEF-03 present in the virus suspension used for the i.c. inoculation of monkeys and the two viruses recovered from monkeys (R5 and Q15). The four viruses were identical even at positions known to be heterogeneous $[13,20]$ except for positions 9337 , 9661 and 10,675 in which one or the other base already detected became the predominant nucleotide according to the virus considered (Table 4). None of these heterogeneities led to amino acid changes.

Alterations in plaque size can also arise as a consequence of selection of viruses that harbor mutations. Therefore, we

Table 3

Thermostability of 17DD virus produced in primary CEF cultures

\begin{tabular}{|c|c|c|c|c|c|c|c|}
\hline \multirow[t]{2}{*}{ Experiment } & \multirow[t]{2}{*}{ Presentation } & \multicolumn{6}{|c|}{ Titers $^{\mathrm{a}}$} \\
\hline & & Bulk & At filling & After filling & $-70^{\circ} \mathrm{C}$ & $37^{\circ} \mathrm{C} / 14$ days & Loss \\
\hline \multirow[t]{3}{*}{01} & 5-Dose YFCEF-06 & 6.11 & 5.6 & 5.11 & 4.24 & 3.98 & 0.26 \\
\hline & 50-Dose & 6.69 & 6.6 & 6.5 & 4.65 & 4.08 & 0.57 \\
\hline & Vaccine control & - & - & - & 4.97 & 4.37 & 0.6 \\
\hline \multirow[t]{3}{*}{02} & 5-Dose YFCEF-05 & 5.56 & 5.49 & 5.07 & 4.13 & 3.95 & 0.18 \\
\hline & 50-Dose & 6.32 & 5.95 & 6.1 & 4.23 & 3.78 & 0.45 \\
\hline & Vaccine control & - & - & - & 4.69 & 4.22 & 0.47 \\
\hline \multirow[t]{3}{*}{03} & 5-Dose YFCEF-07 & 5.68 & 5.36 & 5.2 & 4.54 & 4.15 & 0.39 \\
\hline & 50-Dose & 6.32 & 6.17 & 6.05 & 4.32 & 3.84 & 0.48 \\
\hline & Vaccine control & - & - & - & 4.75 & 4.23 & 0.52 \\
\hline
\end{tabular}

${ }^{\mathrm{a}}$ All titers are expressed in $\log _{10} \mathrm{PFU} / \mathrm{dose}$. 
Table 4

Nucleotide sequence analysis of 17DD virus produced in primary CEF cultures

\begin{tabular}{cllllll}
\hline Position $^{\mathrm{a}}$ & Gene $^{\mathrm{a}}$ & YFCEF-03 & YFCEF-03 Q15 & YFCEF-03 R5 & 102/84 & Amino acid change \\
\hline 1003 & $\mathrm{E}$ & $\mathrm{T} / \mathrm{C}$ & $\mathrm{T} / \mathrm{C}$ & $\mathrm{T} / \mathrm{C}$ & $\mathrm{T} / \mathrm{C}$ & - \\
4523 & NS2B & T/C & T/C & T/C & C/T & - \\
6673 & NS4A & C/T & C/T & C/T & C/T & - \\
9337 & NS5 & A/G & $\mathrm{A}$ & G/A & A & - \\
9661 & NS5 & T & T & C/T & T & - \\
9988 & NS5 & C/T & C/T $/ T$ & C/T & - \\
10174 & NS5 & A/G & A/G & A/G & A/G & - \\
10675 & 3'-UTR & A & A/G & A/G & A/G & - \\
\hline
\end{tabular}

${ }^{\text {a }}$ Rice et al [14].

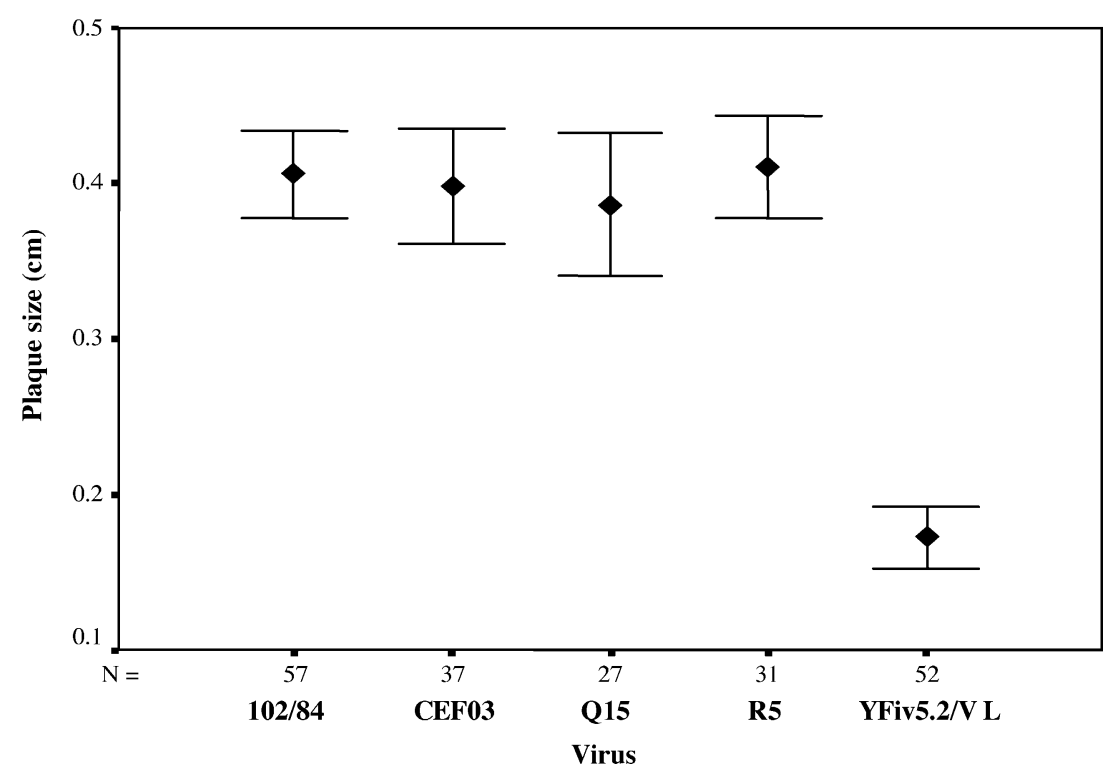

Fig. 1. Plaque size analysis of YF 17DD virus. The 17DD viruses were plaqued on Vero cell monolayers $\left(100,000 \mathrm{cells} / \mathrm{cm}^{2}\right)$. YF 17DD 102/84 virus corresponds to the working seed lot virus, YFCEF-03 is one of the vaccine batches produced in CEF; Q15 and R5 are viruses isolated from viremic monkeys which received YFCEF-03 virus; YFiv5.2/VL is a small plaque size virus derived from cloned cDNA (Marchevsky et al. [17]). The total number of plaques that were measured to estimate the size is shown above the virus name. Size is displayed in centimeter. The values above are the average of three separate experiments.

have examined the plaque size phenotype as an additional evidence for the genetic stability of the virus passaged in primary CEF culture and monkeys. The YF 17DD virus has a heterogeneous, but predominantly large plaque phenotype in Vero cells (about $4.0 \mathrm{~mm}$ ) whereas the cDNA-derived YFiv5.2/VL virus [17] produces significantly smaller plaques (mean of $1.5 \mathrm{~mm}$; Fig. 1). The virus present in the YFCEF-03 batch and the two viruses recovered from viremic monkeys retained the large plaque phenotype (Fig. 1) characteristic of YF 17DD virus.

\subsection{Attenuation of the experimental virus}

\subsubsection{Mouse neurovirulence}

Although there is no clear correlation between mouse neurovirulence and virulence or attenuation of $\mathrm{YF}$ viruses with clinical events in humans, it was relevant to demonstrate that the CEF-passaged 17DD virus approximates in neurovirulence to parental virus $\mathrm{YF}$ 17DD, mainly because YF 17D virus retains a certain degree of neurotropism and neurovirulence for

Table 5

Neurovirulence for mice of YF 17DD viruses

\begin{tabular}{lllll}
\hline Virus & Dose $\log _{10}$ PFU & \% Mortality $($ dead/test) & Average survival time & $\log _{10}$ PFU/MLD $_{50}$ \\
\hline 17DD (vaccine) & 3.28 & $80(8 / 10)$ & $9.90 \pm 1.30$ & 1.15 \\
17DD-CEF & 3.18 & $90(9 / 10)$ & $10.22 \pm 1.23$ & 1.46 \\
R5 $^{\mathrm{b}}$ & 3.08 & $100(10 / 10)$ & $10.40 \pm 1.14$ & 1.46 \\
Q15 $^{\mathrm{b}}$ & 3.48 & $100(10 / 10)$ & $11.60 \pm 1.02$ & 1.33 \\
\hline
\end{tabular}

a $95 \%$ confidence interval.

b Viruses isolated from viremic rhesus monkeys. 
Table 6

Parameters of neurovirulence test in rhesus monkeys with YF 17DD 102/84 and YFCEF-03 viruses

\begin{tabular}{|c|c|c|c|c|c|c|c|c|}
\hline \multirow[t]{2}{*}{ Virus } & \multicolumn{2}{|l|}{ Monkeys } & \multicolumn{3}{|c|}{ Viremia $^{a} \log _{10}$ PFU/mL (days post-inoculation) } & \multirow[t]{2}{*}{ Days $T \geq 40^{\circ} \mathrm{C}$} & \multicolumn{2}{|c|}{$\mathrm{PRNT}_{50}{ }^{\mathrm{b}}(\mathrm{mIU} / \mathrm{mL})$} \\
\hline & Number & Sex & Second & Fourth & Sixth & & Pre & Post \\
\hline \multirow[t]{10}{*}{$102 / 84$} & Q1 & M & $<1.40$ & 2.44 & 1.70 & 1 & $<320$ & 37.153 \\
\hline & Q30 & $\mathrm{F}$ & $<1.40$ & 2.18 & $<1.40$ & 7 & $<269$ & 20.417 \\
\hline & Q33 & M & 1.40 & $<1.40$ & $<1.40$ & 2 & $<288$ & 22.909 \\
\hline & Q46 & $\mathrm{F}$ & 2.48 & 2.48 & $<1.40$ & 2 & $<288$ & 38.019 \\
\hline & Q49 & M & 2.18 & 2.18 & $<1.40$ & 3 & $<288$ & 14.125 \\
\hline & $\mathrm{R} 1$ & M & 1.70 & 1.40 & $<1.40$ & 2 & $<323$ & 14.454 \\
\hline & R7 & M & 2.18 & 1.88 & $<1.40$ & 2 & $<323$ & 34.674 \\
\hline & R24 & $\mathrm{F}$ & 1.70 & $<1.40$ & $<1.40$ & 3 & $<162$ & 19.055 \\
\hline & $\mathrm{R} 25$ & M & 1.40 & $<1.40$ & $<1.40$ & 5 & $<162$ & 20.417 \\
\hline & Q28 & $\mathrm{F}$ & $<1.40$ & 1.70 & $<1.40$ & 3 & $<470$ & 33.113 \\
\hline \multirow[t]{10}{*}{ YF CEF-03 } & Q10 & $\mathrm{F}$ & 2.44 & 2.51 & $<1.40$ & 3 & $<320$ & 27.542 \\
\hline & Q15 & M & $<1.40$ & 1.88 & $<1.40$ & 6 & $<692$ & 85.114 \\
\hline & Q26 & $\mathrm{F}$ & $<1.40$ & 1.40 & $<1.40$ & 3 & $<269$ & 138.038 \\
\hline & Q27 & M & 2.80 & 2.10 & $<1.40$ & 3 & $<269$ & 69.183 \\
\hline & Q41 & M & 1.40 & 1.88 & 1.70 & 3 & $<602$ & 63.096 \\
\hline & R5 & M & $<1.40$ & 1.40 & $<1.40$ & 5 & $<323$ & 18.621 \\
\hline & Q14 & $\mathrm{F}$ & 1.70 & 1.88 & $<1.40$ & 2 & $<327$ & 29.512 \\
\hline & R21 & M & 2.51 & $<1.40$ & $<1.40$ & 3 & $<162$ & 20.417 \\
\hline & $\mathrm{R} 23$ & M & 2.30 & $<1.40$ & $<1.40$ & 3 & $<162$ & 12.882 \\
\hline & $\mathrm{R} 44$ & $\mathrm{~F}$ & 2.57 & 2.10 & $<1.40$ & 3 & $<479$ & 47.863 \\
\hline
\end{tabular}

\footnotetext{
${ }^{a}$ Mean peak titer for 17DD 102/84 is 1.93 (S.D. 0.40) and 2.11 (S.D. 0.48) for YF CEF03. The difference between the means is not statistically significant (Kruskal-Wallis test, $p=0.23$ ).

b 17DD 102/84 virus (GMT 25,433); YFCEF-03 virus (GMT 51,226). The difference between the means is not statistically significant (Kruskal-Wallis test, $p=0.17)$.
}

mice and nonhuman primates inoculated by i.c. route [1].

Groups of 3-week-old Swiss Webster mice were inoculated i.c. with serial dilutions of each virus. The mortality rate, average survival time and the ratio PFU/MLD 50 were established (Table 5). In general it was observed that the higher the dose, the higher the mortality rates reaching $80-100 \%$ (data not shown). Table 5 shows the data from the mice that received approximately $3.0 \log _{10}$ PFU. The mortality rates were: $80 \%$ for 17DD (AST of 9.9 days), $90 \%$ for YFCEF-03 virus (AST of 10.2 days), $100 \%$ for R5 (AST of 10.4 days) and $100 \%$ for Q15 (AST of 11.6 days).

Kaplan-Meyer survival curves and the log rank test showed that the difference of average survival times the 17DD group and the YF CEF03 was not statistically significant $(p=0.67)$. Since the distribution of data was skewed, the Kruskal-Wallis non-parametric test was used, and also suggested no significant difference $(T=4.89 ; p=0.21)$. The differences in mortality rates among groups were not statistically significant, either $(p=0.27)$.

\subsubsection{Monkey neurovirulence}

To further confirm that the YF 17DD virus produced in CEF cultures did not undergo phenotypic changes as predicted from the nucleotide sequence data and retained its attenuation we carried out a full monkey neurovirulence test as recommended for YF 17D vaccine [2]. These results are shown in Tables 6 and 7.

\subsubsection{Febrile response}

All animals inoculated i.c. developed fever equal or above $40.0^{\circ} \mathrm{C}$ that lasted from 1 to 7 days (Table 6) with an average of 3 days/monkey for 102/84 virus and 3.4 days/monkey for YFCEF-03.

\subsubsection{Viremia}

The viremia detected in the sera of monkeys during the second, fourth and sixth days post inoculation is shown in Table 6. Circulating virus could be detected in all animals inoculated with either virus. The total number of days of detectable viremia for animals inoculated with 102/84 virus was 16 with a mean peak titer of $1.93 \log _{10} \mathrm{PFU} / \mathrm{mL}$. For YFCEF-03 virus, the number of viremic days was somewhat lower (10 days) and the mean peak titer was not significantly different $\left(2.11 \log _{10} \mathrm{PFU} / \mathrm{mL}\right)$. The use of the nonparametric test of Kruskal-Wallis to analyze the difference between peak viremias for both groups failed to reveal any statistically significant difference $(p=0.23)$.

\subsubsection{Clinical findings}

Three animals (Q1, Q33 and Q49) inoculated with 17DD $102 / 84$ seed virus developed at least 1 day of clinical encephalitis (grade 3) and the group mean was 0.43 (S.D. 0.41) (Table 7). Five animals that received YFCEF-03 developed grade 3 scores for at least 1 day (Q15, Q26, Q27, Q41 and R23) yielding a group mean of 0.83 (S.D. 0.71; Table 7). Although the means are very different, the non uniform dis- 
Table 7

Comparison of clinical and histological scores of rhesus monkeys inoculated with YF 17DD 102/84 and 17DD YFCEF-03

\begin{tabular}{|c|c|c|c|c|c|}
\hline Virus & Animals & Clinical score $^{\mathrm{a}}$ & Discriminator areas & Target area & Combined score ${ }^{b}$ \\
\hline \multirow[t]{12}{*}{$102 / 84$} & Q1 & 0.57 & 0.64 & 1.00 & 0.82 \\
\hline & Q30 & 0.30 & 0.69 & 2.00 & 1.34 \\
\hline & Q33 & 0.94 & 0.53 & 1.00 & 0.76 \\
\hline & Q46 & 0.34 & 0.67 & 2.00 & 1.33 \\
\hline & Q49 & 0.74 & 0.72 & 2.00 & 1.36 \\
\hline & $\mathrm{R} 1$ & 0.03 & 0.57 & 2.00 & 1.28 \\
\hline & R7 & 0.00 & 0.09 & 1.00 & 0.54 \\
\hline & $\mathrm{R} 24$ & 0.03 & 0.35 & 1.50 & 0.92 \\
\hline & $\mathrm{R} 25$ & 0.20 & 0.63 & 2.00 & 1.31 \\
\hline & Q28 & 1.20 & 0.76 & 1.00 & 0.88 \\
\hline & Mean & 0.43 & 0.56 & 1.55 & 1.05 \\
\hline & S.D. & 0.41 & 0.20 & 0.49 & 0.30 \\
\hline \multirow[t]{12}{*}{ YFCEF-03 } & Q10 & 0.50 & 1.14 & 2.0 & 1.57 \\
\hline & Q15 & 2.50 & 1.96 & 3.0 & 2.48 \\
\hline & Q26 & 0.97 & 1.54 & 2.0 & 1.77 \\
\hline & Q27 & 0.24 & 0.70 & 2.0 & 1.35 \\
\hline & Q41 & 1.60 & 1.90 & 1.5 & 1.70 \\
\hline & R5 & 0.30 & 0.26 & 1.0 & 0.63 \\
\hline & Q14 & 0.30 & 0.48 & 2.0 & 1.24 \\
\hline & R21 & 0.60 & 0.80 & 2.0 & 1.40 \\
\hline & R23 & 0.90 & 0.47 & 2.0 & 1.23 \\
\hline & $\mathrm{R} 44$ & 0.47 & 0.42 & 2.0 & 1.21 \\
\hline & Mean & 0.83 & 0.96 & 1.95 & 1.45 \\
\hline & S.D. & 0.71 & 0.63 & 0.49 & 0.48 \\
\hline
\end{tabular}

${ }^{\text {a }}$ Kruskal-Wallis test, $p=0.15$. Excluding monkey Q15 from the CEF03 virus group, the mean clinical score drops to 0.63 (Kruskal-Wallis test, $p=0.25$ ).

b Mann-Whitney test with or without monkey Q15, the means for the combined histological scores are not different $(p=0.065$ and 0.113 , respectively).

tribution of the clinical scores is indicated by the high values for the standard deviation in both groups making it difficult to detect a difference as statistically significant. Using the nonparametric Kruskal-Wallis test, given the assimetry of the data, it did not reject the null hypothesis that both groups are equal $(p=0.15)$. If we exclude monkey Q15, means are 0.43 for $102 / 84$ and 0.63 for YF CEF instead of 0.83 . The use of the nonparametric test revealed no statistically significant difference between the two groups $(p=0.25)$.

\subsubsection{Histological findings}

All 20 rhesus monkeys inoculated i.c. with either virus developed histological lesions in the CNS (Table 7). No lesions were observed in liver, kidney, adrenals, heart, spleen or lungs (data not shown).

As proposed by Levenbook et al. [21] the target area in rhesus monkeys CNS for several vaccine viruses is the $s$. nigra. In this study, the $s$. nigra presented the highest histological scores for both viruses. Based on the individual values shown in Table 7, 102/84 virus had an average score in this area of 1.55 with 1.95 for YFCEF-03 virus. In a total of 49 monkeys that underwent neurovirulence tests with 17DD $102 / 84$ seed lot the average target area score was 1.52 (S.D. 0.55 ; [12]). Among the discriminator areas in the CNS, the putamen, g. pallidus and $n$. caudatus were the areas most affected. Monkey Q46 inoculated with 102/84 virus did not show any specific neurological signs and presented grade 3 lesion on one hemisphere of the n. caudatus. Four monkeys
(Q10, Q15, Q26 and Q41) inoculated with YFCEF-03 virus developed grade 3 lesions in the $n$. caudatus,putamen and $g$. pallidus (in at least one of these areas). Monkey Q15 died on day 14 post inoculation whereas all the others survived. Monkey Q10 showed no specific signs of encephalitis but monkeys Q26 and Q41 did for 3 and 8 days, respectively. Monkey Q15 showed grade 3 in both hemispheres of the $s$. nigra and several areas of the cervical and lumbar enlargements whereas monkey Q41 showed grade 4 lesions in one hemisphere of each of the three CNS structures (n. caudatus, putamen and g. pallidus). This animal was the only one from among 145 rhesus monkeys that our group has inoculated with 17D viruses that presented a discriminator area score higher than a target area score.

The average discriminator area score for 102/84 virus was 0.56 (S.D. 0.2) and 0.96 (S.D. 0.63) for YFCEF-03 virus. Histological examination of the target and discriminator areas provides a complete assessment of neurovirulence of the test product [21]. For 102/84 virus the score from discriminator plus target areas (combined score) was 1.05 and for YFCEF-03 virus it was 1.45 . The values for the combined scores in five complete tests with $102 / 84$ virus varied between 0.96 and 1.37 with an average of 1.07 [11]. We used the Mann-Whitney test to verify the significance of the difference between the means for the histological scores given the nonsymmetrical distribution of values. Such analyses, with or without monkey Q15, revealed no significant differences ( $p=0.065$ and 0.113 , respectively). The behavior of the two 


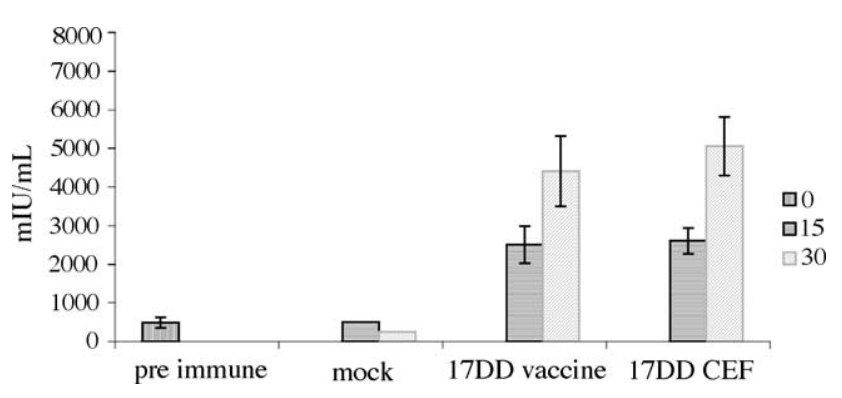

Fig. 2. Immunogenicity of 17DD-CEF virus in mice. Two groups of 16 mice each received a single dose of $5 \log _{10}$ PFU and were bled at various times (0-15-30 days). A third group with eight animals was mock infected with culture medium only. The pre-immune sera represent samples from two animals of each group (mock, 17DD vaccine and 17DD CEF). Neutralization titers were derived on individual serum for each animal except for the group receiving medium only (mock) for which the animal sera were pooled. The error bar represents confidence limits (95\%).

viruses in the monkey neurovirulence test reached borderline level of statistical significance.

\subsubsection{Immunogenicity}

We compared the immunogenicity of the CEF culturepassaged 17DD virus and the 17DD vaccine produced in chick embryos by subcutaneous inoculation of mice and analysis of seroconversion by PRNT. As compared to the pre-immunisation values we observed an increase of PRNT titers of eight-fold for both YF 17DD and 17DD YFCEF-03 viruses at 30 days after immunization whereas 199 mediuminoculated mice did not display any neutralising antibodies to YF virus (Fig. 2). At day 45, all three groups were challenged with 4000 PFU by the intracerebral route with the chicken embryo produced 17DD vaccine virus. The group that received medium showed a mortality of $83.3 \%$ (AST 9.83 \pm 1.27 ) whereas all animals that received either the 17DD vaccine or YF CEF-03 virus were protected. These results further suggested the appropriate immunogenicity of the experimental CEF virus.

Although intracerebral inoculation of monkeys is not the preferred route to examine the immunogenicity of an experimental virus, the sera from monkeys that received the YF 17DD vaccine and YFCEF-03 viruses were analyzed for the presence of neutralizing antibodies. Table 6 shows that all 20 monkeys developed neutralizing antibodies to YF based on PRNT $_{50}$ assays. The titers varied from $14,125 \mathrm{mIU} / \mathrm{mL}$ to 38,019 for 102/84 virus (GMT 25,433) and from 12,882 to
138,038 mIU/mL for YFCEF-03 virus (GMT 51,226). This difference between the means of both groups was analysed by the $t$ test which indicated borderline statistical significance $(p=0.06)$ but given that the data is assymetrical and variances are not homogeneous the nonparametric Kruskal-Wallis test was also used for the analysis. This test did not rejected the null hypothesis, that is, both groups are equal $(p=0.17)$.

\section{Discussion}

Development of a method to produce YF vaccine virus in tissue culture would help to improve our ability to efficiently manufacture this important product. Here we describe a highly efficient and economic process for production of $\mathrm{YF}$ 17DD virus in CEF cell culture. The process has been applied to the production of experimental vaccines from several substrains of 17D virus, including a virus derived from cloned complementary cDNA through infectious clone technology.

The process was established using YF 17DD 102/84 virus, our secondary seed lot that is currently being used for largescale human vaccine production. The experimental viruses produced in primary CEF cultures are, therefore, at the same passage level as the current vaccine. One key concern in the use of an alternative growth substrate for vaccine virus production is that genetic variants may be selected due to different pressure posed by this new cell system when compared to the environment in ovo. Although we felt that the probability of selecting a virulent derivative of YF 17DD in the course of a single passage cell culture was low we completely sequenced one of the experimental vaccine batches revealing no significant changes in the majority genetic population.

Another important aspect of selection of a vaccine growth substrate relates to yields. Table 8 shows a comparative analysis of yields in primary CEF cultures and chick embryos with regard to several parameters. The most important point of this table is that application of the CEF methodology results in the production of a much higher number of doses per egg. Together with the low consumption of working seed lot virus and the observed thermostability of the resulting product, it is evident that producing YF vaccines in CEF cultures is now feasible.

Using our CEF methodology, vaccine lots also contain less chicken embryo protein. YF 17D vaccine produced in CEF culture has a much lower concentration of protein

Table 8

Relative yield of 17D virus from whole embryos and embryo cell cultures

\begin{tabular}{lll}
\hline Parameter & Production in chick embryos & Production in CEF \\
\hline Yield/egg & $7.43 \log _{10}$ PFU (number of doses 300$)$ & $10.05 \log _{10}$ PFU (number of doses 120,000$)$ \\
Yield/vial of seed stock & $10.04 \log _{10}$ PFU & $10.55 \log _{10}$ PFU \\
Volume of embryo or single harvest (S.H.)/embryo & $\cong 3.0 \mathrm{~mL}$ & $\cong 1000 \mathrm{~mL}$ \\
Mean titre of S.H. & $\cong 7.00 \log _{10} \mathrm{PFU} / \mathrm{mL}$ & $\cong 7.02 \log _{10} \mathrm{PFU} / \mathrm{mL}$ \\
Protein nitrogen & $\cong 25 \mu \mathrm{g} / \mathrm{HD}$ & $\cong 0.35 \mu \mathrm{g} / \mathrm{HD}$ \\
\hline
\end{tabular}

a HD: human dose. 
$(0.35 \mu \mathrm{g} / \mathrm{HD}$; Table 8$)$ than the embryo-produced vaccine ( $\cong 25 \mu \mathrm{g} / \mathrm{HD}$ ). It is likely that the lower protein content of the CEF product will lead to less frequent adverse events due to allergic reactions.

One key aspect for validating the CEF propagation procedure to allow it to replace the current methodology is to demonstrate that the CEF-produced virus retains the attenuation displayed by the secondary seed virus. In mice, the secondary seed virus and the experimental virus are indistinguishable. To further compare these viruses, we analyzed their phenotype by i.c. inoculation of 10 monkeys with each virus according to $\mathrm{WHO}$ guidelines.

These studies revealed that all monkey viremias were brief and low-level as indicated by mean peak titers and mean duration. The definition put forward by [22] of viscerotropism of 17D virus limits the amount of circulating virus to below 500 mouse $\mathrm{LD}_{50} / 0.03 \mathrm{~mL}$ for all (10 out of 10) sera and $\geq 100 \mathrm{LD}_{50} / 0.03 \mathrm{~mL}$ in one out of 10 monkey sera at 1:10 dilution. In this regard, the highest viremia observed $\left(2.80 \log _{10} \mathrm{PFU} / \mathrm{mL}\right.$ for monkey Q27, inoculated with YFCEF-03 virus), corresponds to $3.78 \mathrm{LD}_{50} / 0.03 \mathrm{~mL}$, therefore well below the established limits. In addition, the range of titers we observed in our 20 monkeys were similar to those observed for rhesus monkeys inoculated with attenuated 17D/JE SA-14-14-2 and 17D-den2 chimeric viruses and YF 17D-204 virus [23-25].

The use of the nonparametric Kruskal-Wallis test to analyze the difference between peak viremias for both groups failed to reveal any statistically significant difference $(p=0.23)$ suggesting the viruses replicate to the same extent in the monkey CNS.

This difference between the means of neutralizing antibody titers for monkeys inoculated with YF 17DD 102/84 or CEF03 was analyzed by the $t$ test $(p=0.06)$ and the nonparametric Kruskal-Wallis $(p=0.17)$. Both failed to reveal any significant difference although the former suggests there may be a difference in their capability for eliciting antibodies.

The attenuation of YF 17D vaccine viruses is traditionally assessed from clinical evidence of encephalitis and by evaluation of histological lesions after intracerebral inoculation of 10 monkeys with an experimental vaccine virus and a characterized, safe 17D virus. According to WHO criteria [8] the mean clinical score of the monkeys injected with the experimental virus shall not exceed the mean clinical score of the monkeys that received the reference vaccine virus. The group mean score for our experimental YF CEF-03 virus was 0.83 , almost twice as high as the 17DD 102/84 virus score of 0.43 . The extreme variability of clinical scores among monkeys in both groups is indicated by the high values for the standard deviation. It must be noted that Marchevsky et al. [11] have found that clinical scores do have some variability from test to test with an interval of $0-0.58$ ( $n=49$ animals). However, even when we have removed one animal (Q15) that produced particularly high clinical score from our YFCEF-03 analyses the mean clinical score for the YFCEF-03 experimental vaccine would still fall outside the interval established
(0.65) suggesting a higher neurovirulence for this test virus. These differences, however, were not found to be statistically significant. A nonparametric test also failed to reveal a significant difference between the two groups (17DD 102/84 versus CEF $03 ; p=0.15$ or 0.25 , with or without monkey $\mathrm{Q} 15$, respectively).

For the histological criterion of the neurotropism to be satisfied the mean combined score for monkeys that received the experimental preparation shall not be significantly greater (at the 5\% significance level) than the overall mean score for the monkeys injected with the reference virus [2]. The frequency and severity of lesions were greater in animals which received the experimental YF vaccine (CEF03) than in those given the control vaccine (17DD 102/84). The averages for each virus were found to be different $(t$ test; $p=0.037$ ) suggesting a higher neurovirulence for the experimental virus. If the score for monkey Q15 is excluded the difference between the means is reduced to a borderline level of significance $(p=0.067)$.

Considering that a nonsymmetrical distribution of values was noted, a non-parametric test would be more appropriate to verify the significance of the difference between the means for the histological scores. Using the Mann-Whitney test with or without monkey Q15, the means for histological scores are not different ( $p=0.065$ and 0.113 , respectively). Thus, we have concluded that the difference between the two viruses in the monkey neurovirulence test reached borderline level of statistical significance.

It is noteworthy that the viruses recovered from two monkeys (R5 and Q15) turned out to be genetically identical to the original 102/84 parental virus despite the difference in clinical manifestations observed during the MNVT.

It is important to underscore the limitations of genome analysis in this study. A limitation is that yellow fever 17DD vaccine is not biologically cloned, and is known to be a mixture of multiple sub-populations of virions reflecting the quasi species nature of flaviviruses. Molecular analysis results in a consensus sequence and is relatively insensitive for the demonstration of minority virus sub-populations. Only when present at a concentration of $>10-20 \%$, will such subpopulations be detected. It is generally accepted that selection of a mutation responsible for an increased virulence, leading to an exarcebated infection would result in the altered genotype being the majority species in the consensus sequence. A second assumption is that one or more amino acid changes would be required for a significant change in virulence, although it is theoretically possible that silent changes could alter secondary structure and biological properties of the virus.

A comparison of the nucleotide sequences for the secondary 17DD 102/84 seed, the YF CEF03 vaccine batch and the viruses recovered from the two monkeys revealed only heterogeneities, that is, two nucleotides were detected by sequencing. This suggests the coexistence of viral populations bearing one or the other nucleotide at that particular position. When a particular heterogeneity was not observed in one virus, one of the nucleotides of that heterogeneous posi- 
tion was always present none of which resulted in an amino acid change (Table 4). Therefore, these variations cannot be considered mutations and related to the increased clinical and histological scores such as observed for monkey Q15.

It is noteworthy that cases of severe adverse events associated with the 17D vaccine have been reported [26-28] but the genetic make-up of each host was held responsible for such rare events as no genetic changes could be associated with the viruses [13]. It may be relevant to that usually one animal in each group of ten develops more severe forms of encephalitis following i.c. inoculation of YF 17DD 102/84 virus and that may also reflect individual factors. The frequency may be higher in this case due to the use of the intracerebral route.

As discussed above for the histological scores the averages for each virus 17DD 102/84 and 17DD CEF03 were found to be different. However, if the score for monkey Q15 was excluded the difference between the means is reduced to a borderline level of significance or no significance if a nonparametric test was used given the data assymetrical distribution. Therefore, the slightly higher neurovirulence score assigned to YF 17DD virus CEF03 may be a consequence of individual responses to the virus, in particular monkey Q15, which showed the highest clinical and histological scores. This view would be supported by the absence of significant nucleotide sequence alterations in the virus recovered from this animal.

The cell culture-derived vaccine is expected to significantly increase vaccine viral yields, thereby reducing costs. Although improvement of manufacture of 17D vaccine in cell culture has been a goal for many years previous attempts have failed because of alterations in the virulence phenotype during the passage in cell culture or poor yields due to in vitro interferon production [1]. We believe that the data shown here indicate it is possible to produce YF 17D virus in CEF cultures with yields compatible with large scale vaccine production. Our genetic and in vitro testing demonstrated the stability of the CEF-produced virus. Specifically, parameters such as plaque size, viremia and immunogenicity in monkeys and attenuation and immunogenicity for mice in addition to complete nucleotide sequence of the viral genome have suggested that the 17DD virus produced in CEF is indistinguishable from the original seed lot virus. However, the results from monkey neurovirulence tests indicated a somewhat higher monkey neurovirulence score. Since these findings could represent test-to-test variation, we feel that they warrant further studies on the attenuation for monkeys of 'other CEF-derived vaccine lots.

\section{Acknowledgements}

We thank the Instituto de Tecnologia em Imunobiológicos (Bio-Manguinhos) for the continuous interest and support to this work. We also thank Elena Caride and Peter W. Mason for critical reading of the manuscript. Financial support from FIOCRUZ (PAPES) and CNPq is greatly acknowledged.

\section{References}

[1] Monath TP. Yellow fever. In: Plotkin SA, Orenstein WA, editors. Vaccines. Third ed. USA: W.B. Saunders Company; 1999. p. 815-998.

[2] WHO. Requirements for yellow fever vaccine. WHO Tech Rep Ser 1998;872:31-68.

[3] Hussain AI, Johnson JA, Da Silva Freire M, Heneine W. Identification and characterization of avian retroviruses in chicken embryoderived yellow fever vaccines: investigation of transmission to vaccine recipients. J Virol 2003;77(2):1105-11.

[4] Pan American Health Organization. Modernization of yellow fever production. Washington, DC: PAHO; 1980.

[5] Lopes OS. Desenvolvimento da vacina contra a febre amarela em cultura de tecido. Simpósio Internacional sobre febre amarela e dengue. Cinqüentenário da Introdução da cepa 17D no Brasil; 1988. p. 253-9.

[6] Marchevsky RS. Resultados de Neurovirulência. Simpósio Internacional sobre febre amarela e dengue. Cinqüentenário da Introdução da cepa 17D no Brasil; 1988. p. 247-52.

[7] Rubim H. Chick embryo cells. In: Kruse PF, Patterson MK, editors. Tissue culture methods and application. New York: Academic Press; 1973. p. 119-23.

[8] WHO. Requirements for mumps vaccine (Live). WHO Tech Rep Ser 1987;760:139-58.

[9] Post PR, Carvalho R, Freire MS, Galler R. The early use of yellow fever virus strain 17D for vaccine production in Brazil. Mem Inst Oswaldo Cruz 2001;96(6):849-57.

[10] Caufour PS, Motta MCA, Yamamura AMY, Vazquez S, Ferreira II, Jabor AV, et al. Construction, characterization and immunogenicity in mice of recombinant yellow fever 17D-dengue type 2 viruses. Virus Res 2001;79(1-2):1-14.

[11] Marchevsky RS, Freire MS, Coutinho ESF, Galler R. The neurovirulence of yellow fever 17DD vaccine virus to rhesus monkeys. Virology 2003;316(1):55-63.

[12] Stefano I, Sato HK, Pannuti CS, Omoto TM, Mann G, Freire MS, et al. Recent immunization against measles does not interfere with the sero-response to yellow fever vaccine. Vaccine 1999;17(9-10): 1042-6.

[13] Galler R, Pugachev KV, Santos CLS, Ochran SW, Jabor AV, Rodrigues SG, et al. Phenotypic and molecular analyses of yellow fever 17DD vaccine viruses associated with serious adverse events in Brazil. Virology 2001;290(2):309-19.

[14] Rice CM, Lenches E, Eddy SR, Shin SJ, Sheets RL, Strauss JH. Nucleotide sequence of yellow fever virus: implications for flavivirus gene expression and evolution. Science 1985;229(4715):726-33.

[15] Reed LJ, Muench LH. A simple method of estimating fifty percent endpoints. Am J Hygiene 1938;27:493-7.

[16] WHO. The production and testing of the WHO Yellow Fever primary seed virus lot 213/77. Expert Committee on Biological Standardization; 1984. p. 1-61.

[17] Marchevsky RS, Mariano J, Ferreira VS, Almeida E, Cerqueira MJ, Carvalho R, et al. Phenotypic analysis of yellow fever virus derived from complementary DNA. Am J Trop Med Hyg 1995;52(1):75-80.

[18] Drake JW, Holland JJ. Mutation rates among RNA viruses. Proc Natl Acad Sci USA 1999;96(24):13910-3.

[19] Pugachev KV, Guirakhoo F, Ocran SW, Mitchell F, Parsons M, Penal $\mathrm{C}$, et al. High fidelity of yellow fever virus RNA polymerase. J Virol 2004;78(2):1032-8.

[20] Pugachev KV, Ocran SW, Guirakhoo F, Furby D, Monath TP. Heterogeneous nature of the genome of the ARILVAX yellow fever 17D vaccine revealed by consensus sequencing. Vaccine 2002;20(7-8): 996-9.

[21] Levenbook IS, Pellen LJ, Elisberg BL. The monkey safety test for neurovirulence of YF vaccines: the utility of quantitative clinical evaluation and histological examination. J Biol Stand 1987;15(4): 305-13. 
[22] WHO. Expert Committee on Biological Standardization, Requirements for Yellow Fever Vaccine. WHO Tech Rep Ser 1995:154.

[23] Monath TP, Levenbook I, Soike K, Zhang ZX, Rattterree M, Draper $\mathrm{K}$, et al. Chimeric yellow fever 17D-Japanese encephalitis virus vaccine: dose-response effectiveness and extended safety testing in rhesus monkeys. J Virol 2000;74(4):1742-51.

[24] Monath TP, McCarthy K, Bedford P, Johnson CT, Nichols R, Yoksan $\mathrm{S}$, et al. Clinical proof of principle for ChimeriVax: recombinant live, attenuated vaccines against flavivirus infections. Vaccine 2002; 20(7-8):1004-18.

[25] Guirakhoo F, Weltzin R, Chambers TJ, Zhang ZX, Soike K, Ratterree $\mathrm{M}$, et al. Recombinant chimeric yellow fever-dengue type 2 virus is immunogenic and protective in nonhuman primates. J Virol 2000; 74(12):5477-85.

[26] Chan RC, Penney DJ, Little D, Carter IW, Roberts JA, Rawlinson WD. Hepatitis and death following vaccination with 17D-204 yellow fever vaccine. Lancet 2001;358(9276):121-2.

[27] Martin M, Tsai TF, Cropp B, Chang GJ, Holmes DA, Tseng J, et al. Fever and multisystem organ failure associated with 17D-204 yellow fever vaccination: a report of four cases. Lancet 2001;358(9276): 98-104.

[28] Vasconcelos PF, Luna EJ, Galler R, Silva LJ, Coimbra TL, Barros $\mathrm{VL}$, et al. Serious adverse events associated with yellow fever 17DD vaccine in Brazil: a report of two cases. Lancet 2001;358(9276): 91-7. 